\title{
LINES IN A PLANAR SPACE
}

\section{GRATTAN P. MURPHY ${ }^{1}$}

A planar space $P$ is a set $S$ together with a mapping $A$ which attaches to each triple $(p, q, r)$ of points of $S$ a real number $A(p, q, r)$ and which satisfies:

(i) If $p=q$, then $A(p, q, r)=A(p, r, q)=A(r, p, q)=0$ for every $r$.

(ii) For every $p, q, r, s, A(p, q, r) \leqq A(p, q, s)+A(p, r, s)+A(q, r, s)$.

(iii) For any $p, q, r, s$; if $A(p, q, r)=A(p, g, s)=0$, then $p=q$ or $A(q, r, s)=A(p, r, s)=0$.

For convenience we will write $p q r$ for $A(p, q, r)$ for the remainder of the paper.

The usual example of such a space is the Euclidean $n$-space with the $A$-function interpreted as the area of a triangle with vertices $p, q$, and $r$.

Spaces satisfying (i) and (ii) and a variety of conditions in place of (iii) have been studied by Menger [6], Blumenthal [2], Froda [3], Gähler [5] and Freese and Andalafte [4].

For $a \neq b$ points of $P$ we define $L[a, b]=\{x \mid a b x=0\}$. It follows readily that if $L(a, b)$ and $L(c, a)$ are distinct sets, then $L(a, b) \wedge L(c, d)$ contains at most one point.

If $p \in P$ is not an element of $L(a, b)$, we define a distance for points $x, y$ of $L$ by setting $d(x, y)=p x y$.

If $x=y$, then $p x y=0$, but $d(x, y)=p x y=0$. If $d(x, y)=0$, then $p x y=0$ and, since $x$ and $y$ belong to $L(a, b), x y a=x y b=0$. Now, if $x \neq y$, applying (iii) to the quadruple $\{p, x, y, a\}$ gives $p x a=p y a=0$. Application of (iii) to the quadruple $\{p, x, y, b\}$ gives $p x b=p y b=0$. Then since $p x a=p x b=0$, we have $p a b=x a b=0$, also from (iii). However, $p a b>0$, since $p$ is not in $L(a b)$. Therefore, it must follow that $x=y$.

Since condition (iii) may be variously applied to any three distinct points of $S$ by letting $s$ and another of the symbols $p, q, r$ denote the same point, it follows that the $A$-function is symmetric. Symmetry of the distance function follows immediately. The tetrahedral inequality applied to $\{p, x, y, z\}$ gives $p x y \leqq p x z+p y z+x y z$. Since $x y z=0$, we have $d(x, y) \leqq d(x, z)+d(y, z)$.

Consequently $d(x, y)$ is a metric for $L(a, b)$. The set $L(a, b)$ with metric $d$ is denoted $M_{p}(a, b)$.

Presented to the Society, January 25, 1967; received by the editors June 10, 1967.

1 This paper represents a portion of the author's dissertation written under the direction of Raymond W. Freese at St. Louis University. 
We will utilize the following definitions.

A point $b$ is said to be between $a$ and $c$ (denoted by $B(a, b, c)$ ) iff $a b c=0, a c x=a b x+b c x$ for every $x$ and $a, b$ and $c$ are distinct.

A planar space $P$ is convex iff for each pair of different points $p$ and $q$ there exists a between point.

A sequence of points $\left\{x_{n}\right\}$ in a planar space $P$ has limit $x$ iff $\lim p x x_{n}$ $=0$ for every $p$ in $P$.

A sequence $\left\{x_{n}\right\}$ in a planar space $P$ is convergent with respect to $(a, b, c)$ iff $a b c>0$ and $\lim a x_{i} x_{j}=\lim b x_{i} x_{j}=\lim c x_{i} x_{j}=0$.

A planar space is complete with respect to $(a, b, c)$ iff for every sequence $\left\{x_{i}\right\}$ convergent with respect to $(a, b, c)$, there exists a point $x$ of $P$ with $\lim x_{i}=x$.

Theorem. If $P$ is a convex space which is complete with respect to $(p, a, b)$, then $M_{p}(a, b)$ is a complete, convex metric space.

If $x$ and $z$ are elements of $M_{p}(a, b)$, then they are elements of $P$ also. From convexity, there exists a $y$ in $P$ such that $B(x, y, z)$ holds. This gives $x y z=0$, so that $y$ is in $M_{p}(a, b)$, and $p x y+p y z=p x z$ which results in $d(x, y)+d(y, z)=d(x, z)$. But, then $y$ is a between point of $x$ and $z$, so that $M_{p}(a, b)$ is convex. If $\left\{x_{n}\right\}$ is a convergent sequence in $M_{p}(a, b)$, then $\lim d\left(x_{i}, x_{j}\right)=0$. But this implies that $\lim p x_{i} x_{j}=0$. Then, since $a x_{i} x_{j}=b x_{i} x_{j}=0$ and $p a b \neq 0,\left\{x_{n}\right\}$ is a convergent sequence with respect to $(p, a, b)$. But $P$ is complete with respect to $(p, a, b)$ so there is an $x$ in $P$ which is the limit of $\left\{x_{n}\right\}$. From $a b x_{i}=0$ for every $i$, it follows that $a b x=0$ and that $x$ is in $M_{p}(a, b)$, which is, therefore, complete.

A subset $S$ of a planar space $P$ is said to be $A$-congruent with a subset $S^{\prime}$ of a planar space $P^{\prime}$ (denoted $S \equiv S^{\prime}$ ) iff there exists a 1-1 mapping of $S$ onto $S^{\prime}$ such that $p q r=p^{\prime} q^{\prime} r^{\prime}$, where $p^{\prime}, q^{\prime}, r^{\prime}$ are the images of $p, q$, and $r$.

CoROLlaRy. If each four points of $P$ are $A$-congruent with four points of $E_{3}$, then $M_{p}(a, b)$ is congruently contained in $E_{1}$.

Let $x, y, z \in L(a, b)$ and be distinct points. Then $x y z=0$. If each of the four points of $P$ are $A$-congruently contained in $E_{3}$, then there exist $p^{\prime}, x^{\prime}, y^{\prime}$, and $z^{\prime}$ in $E_{3}$ with $p^{\prime} x^{\prime} y^{\prime}=p x y, p^{\prime} x^{\prime} z^{\prime}=p x z, p^{\prime} y^{\prime} z^{\prime}=p y z$ and $x^{\prime} y^{\prime} z^{\prime}=x y z=0$. Consequently $x^{\prime}, y^{\prime}$, and $z^{\prime}$ are collinear and $p^{\prime}, x^{\prime}, y^{\prime}$, and $z^{\prime}$ are coplanar. It follows that one of the points $x^{\prime}, y^{\prime}$, or $z^{\prime}$ is a between point of the other two. Let $y^{\prime}$ be the between point. Then $p^{\prime} x^{\prime} z^{\prime}=p^{\prime} x^{\prime} y^{\prime}+p^{\prime} y^{\prime} z^{\prime}$ from which $p x z=p x y+p y z$ follows. But this gives $d(x, z)=d(x, y)+d(y, z)$ so that the three points are embed- 
dable in $E_{1}$. There are more than four distinct points in $M_{p}(a, b)$ since $a \neq b$ and $M_{p}(a, b)$ is convex.

Since every semimetric space containing more than four points and having the property that each three of its points are embeddable in $E_{1}$ is embeddable in $E_{1}[1], M_{p}(a, b)$ is congruently contained in $E_{1}$.

\section{BIBLIOGRAPHY}

1. L. M. Blumenthal, Theory and applications of distance geometry, Clarendon Press, Oxford, 1953.

2. - Distance geometries, Univ. of Missouri Studies, 13, 1938, no. 2.

3. A. Froda, Espaces p-metrique et leur topologie, C. R. Acad. Sci. Paris 247 (1958), 849-852.

4. R. W. Freese and E. Z. Andalafte, A characterization of 2-betweenness in 2-metric spaces, Canad. J. Math. 18 (1966), 963-968.

5. S. Gähler, 2-metrische Räume und ihre topologische Struktur, Math. Nachr. 26 (1963), 115-148.

6. K. Menger, Untersuchungen über allgemeine Metrik, Math. Ann. 100 (1928), 75-163.

University of MaINE 